\title{
Multianálisis de las organizaciones más valoradas para el desempeño laboral en España
}

\author{
FERREIRO-SEOANE, FRANCISCO JESÚS \\ Departamento de Economía Aplicada \\ Universidad de Santiago de Compostela \\ Correo electrónico: franciscojesus.ferreiro@usc.es
}

\begin{abstract}
RESUMEN
El presente artículo trata de conocer el perfil de las empresas más valoradas para el desempeño laboral en España y determinar la posible influencia que tienen en la valoración total. Para ello, se parte del ranking de las 100 empresas con mayor valoración publicado anualmente por la Revista de Actualidad Económica en el período 2013-2016. En un primer paso, se realiza un análisis descriptivo de los promedios de valoración de las empresas agrupándolas por su nacionalidad, la región en la que tienen su sede, el sector económico, el tamaño y su cotización en el mercado bursátil. En un segundo paso, mediante modelos de regresión múltiple, se tratará de establecer si estas características influyen en la valoración total. A pesar de los estudios realizados por diversos autores, no existe ninguno que analice diversas variables objetivas de las empresas para conocer sus perfiles y su posible influencia en el valor total. Los principales hallazgos serían que la mayoría de las empresas más atractivas para trabajar tienen carácter internacional (17 países); predominan los sectores financieros y actividades profesionales, científicas; ubicadas principalmente en la capital del país (76,8\%); de gran tamaño y que cotizan en bolsa. Se concluye que sólo el tamaño y la cotización en bolsa, influyen en la valoración total de las empresas. Destacamos como principales contribuciones, la novedad de este tipo de estudios a través de métodos cuantitativos y un mayor conocimiento sobre el perfil de las empresas más atractivas para trabajar en España.
\end{abstract}

Palabras clave: empresas, recursos humanos, nacionalidad, territorio, comunidades autónomas.

Clasificación JEL: J24; J28; M51.

MSC2010: 60E05; 60A05. 


\title{
Multi analysis of most valued companies for labour performance in Spain
}

\begin{abstract}
The present article intends to study the profile of the most valued companies regarding their labour performance in Spain. It also tries to determine the influence that this profile's characteristics have on the overall rating. For that purpose, we analyse the 100 companies, taken from the ranking published by the Journal of Actualidad Económica (RAE in Spanish), with the highest value every year between 2013 and 2016. As first step, a descriptive analysis of the averaged valuations of the companies is made, grouping them by their nationality, the regions where the companies have their headquarters, their economic sector, their size and their market/share price. Secondly, using multiple regression models, the influence of these characteristics (as independent variables) on the total value (as a dependent variable) will be analyzed and quantified. Despite the studies carried out by some authors, none of them has analyzed objective variables of the companies to know their profiles and their possible influence on the total value. The main findings show that the majority of the most attractive companies have an international character (17 countries); also, that the predominant sectors are the financial ones and those that develop professional and scientific activities, located in the capital of the country $(76.8 \%)$ with a big size and listed on the market. It concludes that only the size and the market/share price could influence the total valuation of the companies. We highlight as main contributions the newness of this type of study using quantitative methods and a higher knowledge of the profile of the most attractive companies to work for in Spain.
\end{abstract}

Keywords: companies, human resources, nationality, territory, autonomous community. JEL classification: J24; J28; M51.

MSC2010: 60E05; 60A05.

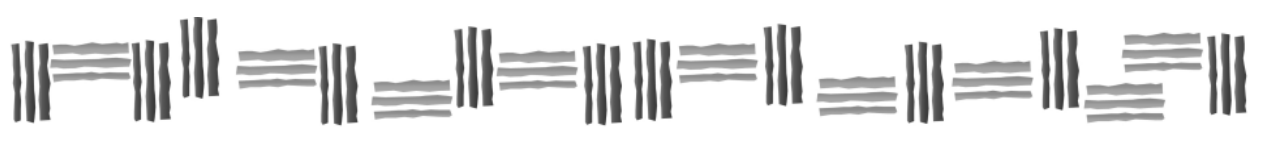




\section{Introducción.}

En enero del 2019, el número de empresas con trabajadores inscritas en la Seguridad Social fue de 1.326.691. Ahora bien, no todas ellas tienen el mismo poder de atracción para los trabajadores. En la actualidad, tener un trabajo es un bien muy apreciado, más si cabe, si la empresa está ubicada en el ranking de las 100 más valoradas para el desempeño laboral en España, como los publicados anualmente por la Revista de Actualidad Económica (RAE).

Dado que no se han encontrado investigaciones que hayan analizado las características de las empresas del ranking publicado por la $R A E \mathrm{u}$ otras, esto constituye una oportunidad para investigar y conocer el perfil de las empresas más valoradas para el desempeño laboral ubicadas en España a través de variables objetivas como son la nacionalidad, la ubicación de su sede central en una comunidad autónoma, la actividad económica de la compañía, el tamaño de la empresa medida por el número de empleados, y la cotización en el mercado bursátil. Por otro lado, también constituye un reto plantear un modelo global de regresión para analizar las posibles influencias de estas variables en las valoraciones obtenidas por las empresas en el ranking de la $R A E$.

Los resultados permitirán conocer el número de empresas españolas en el ranking, si hay empresas anglosajonas, europeas o de otras zonas geo-culturales. A nivel de ubicación regional se podrá determinar en qué zona se encuentran las sedes centrales, si el hecho de que Madrid, en su condición de capital de España, concentra el mayor número de empresas del ranking, si otras zonas desarrolladas del país, como Cataluña o País Vasco, están presentes en el ranking, si existe alguna comunidad autónoma que no alberga ninguna empresa de estas características en su territorio. Otras preguntas académicas que pudieran suscitar interés es conocer a qué se dedican este tipo de empresas, ¿pertenecen al sector sanitario que gestiona recursos humanos de alta talento? ¿Pertenecen al sector energético crucial para la economía española? ¿Se dedican a las actividades financieras y de seguros, sector concentrado y muy competitivo? ¿Cuáles son sus valoraciones? ¿Y el tamaño, tiene relevancia e importancia o es una característica indiferente? Cotizar en el mercado bursátil obliga a las empresas a una serie de requisitos, pero, ¿el hecho de cotizar en dicho mercado influye en una política de recursos humanos con mayor valoración que las que no cotizan?

Para el desarrollo de este trabajo, se empezará con una revisión literaria. En un segundo apartado se definirán los objetivos concretos de esta investigación y la metodología empleada. Posteriormente, partiendo de los datos desglosados de las empresas más atractivas para el desempeño laboral publicado por la Revista de Actualidad Económica, se agregarán variables a cada empresa que definan características de las mismas, se realizará un análisis descriptivo con técnicas estadísticas y se planteará un modelo global de regresión. Se finalizará con un apartado de conclusiones, destacando la importancia de los hallazgos encontrados.

\section{Revisión bibliográfica.}

Según Friedman (2014) y Morgan (2014) las empresas más atractivas para trabajar son aquellas que alcanzan un alto nivel de internacionalización, con un elevado grado tecnológico y que fomentan la movilidad de sus recursos humanos. Este hecho es reflejado en los estudios de Guest (2007) incidiendo en que los profesionales no se limitan a desarrollar su talento en una sola empresa. A su vez el salario no es percibido por los trabajadores como el único elemento a considerar para decantarse por una organización u otra.

Hace más de dos décadas que se publican rankings de las empresas más atractivas para trabajar, con una tendencia a reflejar un centenar, siendo una de las herramientas del branding de los empleadores (Joo \& McLean, 2006). La pretensión es resaltar aquellas organizaciones más valoradas para atraer y retener a un capital humano que se considera como el recurso más valioso de una organización (Joyce, 2003). Este valor intangible es algo fundamental para que las 
empresas puedan ser competitivas, tal como se reflejan en las publicaciones de Hall (1992) y Bonache (1996). Wright, McMahan y McWilliams, (1994) lo definen como el conjunto de capital humano que se encuentra bajo el control de la empresa y con una relación directa de empleo. Así, las propias empresas intentan atraer y retener al talento, hecho que se refuerza con la presencia en alguno de los rankings como una de las mejores empresas para trabajar (Lenaghan \& Eisner, 2006).

Destacando algunos de los principales rankings, tenemos el de la Revista de Actualidad Económica que lo publica todos los años referidos a las empresas que operan en España. Otros internacionalmente conocidos son los que anualmente se publican en EEUU, el Fortune 100 Best Companies to Work for, así como el Glassdoor. En Europa se puede destacar el Best Places to Work. Marcaempleo edita su ranking Merco Talento, que ordena por puntuación a las "100 mejores empresas españolas para trabajar". Universum, elabora rankings de "top employers" para varios países. El Top Employers Institute publica, un listado de empresas certificadas por ellos por "crear condiciones óptimas para el desarrollo de sus empleados, tanto personal como profesionalmente". Por su parte, la revista Workforce elabora un ranking de The World's Top Companies for HR mediante la combinación de varios índices o rankings, entre ellos, el de Great Place to Work.

El estar en alguno de estos rankings redunda positivamente para las empresas que figuran en dicho ranking, tal como reflejan Hinkin y Tracey (2010), que destacan que estar en algún ranking hacen que las empresas se perciban como las mejores en sus prácticas de recursos humanos. En una línea similar son las conclusiones de Fulmer, Gerhart y Scott (2003) y Romero (2004), que resaltan cómo la presencia en algún ranking de las empresas más atractivas para el personal influye sobre las condiciones más idóneas de las mismas para trabajar. Incluso el estar en dichos rankings afecta a las actitudes de los empleados hacia el puesto de trabajo, tal como recogen Ballou, Godwin y Shortridge (2003). Los investigadores científicos usan este tipo de rankings en sus estudios, tal como hicieron Guinot, Chiva y Mallén, (2015), en su trabajo sobre capacidad de aprendizaje organizativo, utilizando entre ellos el de $R A E$.

Un hecho que aumenta las posibilidades de estar en los rankings más atractivos para trabajar, es hacer una buena gestión del talento tal como resalta Alles (2006), la gestión del talento aborda la proyección, el rendimiento y la rotación no deseada. En su trabajo, BethkeLangenegger, Mahler y Staffelbach (2011) afirman que centrarse en retener y desarrollar el talento impacta positivamente en los resultados obtenidos.

El capital humano ha de ser retribuido, siendo esta compensación económica un aspecto que no deben obviar las empresas que buscan la excelencia en la gestión de recursos humanos, tal como reflejaron en sus trabajos Bešlić y Bešlić (2008), que la satisfacción en el trabajo aumentará si los empleados creen que el sistema de recompensas es justo.

Es difícil imaginarse estar en algún ranking de las empresas más atractivas para el desempeño laboral si la organización tiene un mal ambiente de trabajo. Kreps y Spence (1985) sugieren que incrementar el bienestar del empleado aumenta el compromiso de los trabajadores y envía señales a empleados potenciales sobre las condiciones laborales y las normas internas.

Una buena Responsabilidad Social Corporativa $(R S C)$ aumenta las posibilidades de estar presente en estos rankings de reputación que influye positivamente en su valor de mercado (Fernández-Gámez, Gil-Corral \& Galán-Valdivieso, 2016). Kotha, Rajgopal y Rindova (2001) afirman que es un activo inimitable e irremplazable para una empresa.

Finegold y Sosckice (1988) y Betcherman, Leckie y McMullen (1997) concluyen que la toma de decisiones sobre formación es clave en el resultado empresarial y debe ser tenida en cuenta por los directivos, hecho que aumenta las posibilidades de estar incluidos en el ranking. La Teoría del Capital Humano de Mincer (1962) argumenta que la educación y la formación son 
dos formas de inversión complementarias, siendo la formación una inversión rentable (OCDE, 1991). Según Smith (1993) y Osterman (1994) la formación es esencial para la supervivencia de la empresa. Existen evidencias que indican que la excelencia de la organización solo puede alcanzarse si se introducen intervenciones de formación junto con otros factores sociales (Hosie et al., 2013). Además, los empleados satisfechos tienen mayor devoción por su trabajo y son más productivos (Nijhof et al., 1998; Baron, 1991).

\section{Objetivo y metodología.}

\subsection{Objetivo.}

Cada año, la $R A E$ publica una lista de las cien empresas que operan en España más valoradas para el desempeño laboral. El primer objetivo del presente artículo es conocer el perfil y las características de estas empresas, a través de variables que definan su procedencia nacional, la ubicación territorial desde donde dirigen la organización, la actividad de económica, el tamaño y si cotizan en el mercado bursátil. Un segundo objetivo es ver la posible influencia que dichas variables pudieran tener en los valores publicados en el ranking de la Revista de Actualidad Económica.

\subsection{Variables.}

Se han de distinguir tres categorías de variables: las primeras son las usadas por la $R A E$ para la elaboración del ranking (Tabla 1); las segundas son las que definen características de las empresas que están en dicho listado y que se usarán para el análisis descriptivo (Tabla 2); y las terceras (Tabla 3) son una agrupación de las segundas, que nos permite analizar si existe relación entre éstas y los valores obtenidos por las empresas en el ranking de la $R A E$.

\subsubsection{Variables usadas por la $R A E$ para la publicación del ranking}

Tal como se puede apreciar en la Tabla 1, la Revista Actualidad Económica utiliza para la publicación de su ranking seis variables independientes y un total que es la suma de dichas variables.

Tabla 1. Variables usadas por la RAE para valorar las organizaciones más atractivas para el desempeño laboral en España.

\begin{tabular}{|c|c|c|}
\hline Variables & Puntos & $\%$ s/total \\
\hline Gestión del talento (independiente): proyección, rendimiento y rotación no deseada & 240 & $24,0 \%$ \\
\hline $\begin{array}{l}\text { Retribución y compensación (independiente): combinación de salario fijo y } \\
\text { variable, remuneración en especie y beneficios sociales }\end{array}$ & 225 & $22,5 \%$ \\
\hline $\begin{array}{l}\text { Ambiente de trabajo (independiente): horario, teletrabajo, conciliación familiar y } \\
\text { condiciones de trabajo }\end{array}$ & 205 & $20,5 \%$ \\
\hline $\begin{array}{l}\text { Responsabilidad Social Corporativa (RSC) (independiente): políticas sociales y de } \\
\text { voluntariado en las que se involucra a la plantilla }\end{array}$ & 50 & $5,0 \%$ \\
\hline Formación (independiente): inversión recibida por el empleado & 220 & $22,0 \%$ \\
\hline $\begin{array}{l}\text { Empleados (independiente): valoración que tienen los profesionales de la empresa en } \\
\text { la que trabajan }\end{array}$ & 60 & $6,0 \%$ \\
\hline Total (dependiente): corresponde a la suma de las puntuaciones de las dimensiones & 1.000 & $100,0 \%$ \\
\hline
\end{tabular}


La variable independiente, Gestión del talento, puede alcanzar un valor potencial de 240 puntos, que la convierte en la de mayor valor. La gestión del talento de una compañía aumenta la excelencia y refuerza la cultura corporativa, tal como acreditan los trabajos de DiRomualdo, Joyce \& Bression, 2009; Gandossy \& Kao, 2004; y Ashton \& Morton, 2005.

La segunda variable independiente en importancia cuantitativa (225 puntos) del modelo utilizada por la $R A E$ es la retribución y compensación, que evalúa la combinación de salario fijo y variable, la remuneración en especie y los beneficios sociales, tal como indicaron en sus trabajos Brown, 2001; Clark, Kristensen \& Westergard-Nielsen, 2007; y Kabak et al., 2014.

La tercera variable considerada por la RAE para la elaboración del ranking, con una valoración potencial de 205 puntos, es el ambiente de trabajo, pues tal como concluye Helm (2007), crear trabajadores satisfechos, que presenten una buena imagen de la empresa en cualquier interacción con los clientes y otras partes interesadas, aumenta el valor de la empresa. Los empleados son los que crean la reputación de la empresa; a través de ellos, los clientes perciben a la organización (Davies et al., 2003), lo que nos da entender que un mal ambiente de trabajo puede redundar en una mala percepción de la empresa por parte del cliente.

La cuarta variable, ponderada con 50 puntos en el ranking de la $R A E$, es la Responsabilidad Social Corporativa $R S C$. Para Capraro y Srivastava (1997), y Fombrun \& Shanley (1990), la reputación corporativa le confiere a la compañía una ventaja competitiva sostenible y de valor. De acuerdo con Elsbach y Glynn (1996), las empresas deberían comprometer activamente a los empleados en la trasmisión y demostración de la reputación, para mejorar la reputación global de la compañía. Se considera el desarrollo social de las empresas como un elemento importante que forma las percepciones de diferentes partes involucradas y guía sus comportamientos (Arikan et al., 2016).

Existen evidencias que indican que la excelencia de la organización solo puede alcanzarse si se introducen intervenciones de formación (quinta variable del ranking de la $R A E$ y tercera en valor de todas las consideradas) junto con factores sociales como la confianza, la comunicación, el feedback, el tipo de supervisión, la apropiada gestión del desarrollo y el sistema de recompensa, pero también debe tenerse en cuenta el salario como mediador de la satisfacción laboral (Hosie et al., 2013).

Los empleados tienen sus propias percepciones de las empresas en las que trabajan, siendo ésta la sexta variable, con un valor potencial de 60 puntos. La satisfacción global, las recompensas (retribución) y el equilibrio entre trabajo y vida personal/privada influyen en el rendimiento de la empresa, tal y como afirman Kabak et al. (2014). Mejorar la experiencia laboral de los empleados probablemente haga que aumenten sus intenciones de permanecer en la empresa, al igual que la productividad de la misma (Armstrong, 2006; Snyder \& Lopez, 2002).

\subsubsection{Variables usadas en el análisis descriptivo}

Para conocer mejor el perfil de las empresas del ranking, es preciso seleccionar nuevas variables que identifiquen características de estas empresas y permitan hacer análisis. Por ello, una vez publicado el ranking, se ha procedido a construir una matriz de datos a la que se han incorporado nuevas variables exógenas (Tabla 2) como su nacionalidad, la comunidad autónoma en la que tienen su sede social, el número de trabajadores, si cotizan en el mercado bursátil y la actividad principal a la que se dedican, teniendo una base de datos muy amplia que nos permite responder a los objetivos planteados en el artículo a través de análisis estadísticos. 
Tabla 2. Variables incorporadas para el análisis descriptivo de las organizaciones más atractivas para el desempeño laboral en España.

\begin{tabular}{|c|c|}
\hline Variables & Justificación \\
\hline Nacionalidad & $\begin{array}{l}\text { Clasificar la información cuantitativa del ranking por nacionalidad y hacer } \\
\text { análisis estadísticos }\end{array}$ \\
\hline $\begin{array}{l}\text { Ubicación de la sede } \\
\text { central }\end{array}$ & $\begin{array}{l}\text { Conocer dónde están ubicadas las sedes centrales de este tipo de empresas tan } \\
\text { excelentes para los profesionales. ¿Se concentran en una zona determinada de } \\
\text { España o están dispersas por todo el territorio nacional? }\end{array}$ \\
\hline $\begin{array}{l}\text { Actividad } \\
\text { económica }\end{array}$ & $\begin{array}{l}\text { Se busca conocer cuáles son los sectores económicos de este tipo de empresas } \\
\text { y para ello, se clasifican según código CNAE agrupadas al primer dígito }\end{array}$ \\
\hline Tamaño & $\begin{array}{l}\text { Con esta variable se busca saber el tamaño de las empresas que están en este } \\
\text { ranking. ¿Estar en el grupo de privilegio está reservado para las grandes } \\
\text { corporaciones o el tamaño no es un hecho relevante? }\end{array}$ \\
\hline Cotización & $\begin{array}{l}\text { Clasificar las empresas según coticen en el Mercado Secundario de Valores, nos } \\
\text { permitirá conocer mejor el perfil de este tipo de empresas más atractivas para } \\
\text { el trabajar }\end{array}$ \\
\hline
\end{tabular}

Fuente: Elaboración propia.

Nacionalidad. Ibrahim y Shah (2013) habían estudiado la influencia de la nacionalidad de origen en las prácticas de recursos humanos en empresas malayas, concluyendo que no era un aspecto relevante; en cambio Ferner (1997) sí presentó una relación de las diferencias sistemáticas en la gestión de recursos humanos en las empresas multinacionales en función del país de origen. Este hecho fue corroborado posteriormente por Liu (2004) y Guthrie et al. (2008) que documentan los hallazgos en el mismo sentido con investigaciones empíricas más recientes. Por todo ello, se considera oportuno identificar cada empresa con su nacionalidad, lo que permite saber el número de empresas por países, la valoración media, dadas las diferencias de gestión, costumbre y cultura que existen entre las empresas, sus directivos y las políticas empresariales en función del país. En los análisis estadísticos generales se usarán a nivel país, pero en el modelo de regresión lineal múltiple se agruparán a nivel de áreas geo-culturales tales como países anglosajones, Centro y Norte de Europa, Mediterráneo y otros, puesto que, si la usáramos a nivel de país, tendríamos tamaños muestrales irrelevantes desde un punto de vista estadístico. También, dado que existe una presencia relevante de empresas españolas $(38,8 \%)$, se establece una variable dicotómica versus extranjeras.

Ubicación de la sede central. Se encuentran ciertas diferencias entre las prácticas de recursos humanos en distintas zonas detectadas por investigaciones comparativas que emplean diversas técnicas econométricas (Amossé et al., 2016; Conway et al., 2008; Fields, Chan \& Akhtar, 2002; Grimshaw \& Rubery, 2007; Paawee \& Boselie, 2007). Por tanto, otra variable que parece interesante estudiar es dónde están ubicadas las sedes centrales de este tipo de empresas tan excelentes para los profesionales. ¿Se concentran en una zona determinada de España o están dispersas por todo el territorio nacional?, ¿influye esto en la valoración de dichas empresas? En los análisis estadísticos generales se usarán a nivel de Comunidades Autónomas, pero en el modelo de regresión lineal múltiple las agruparemos a nivel de áreas geo-culturales tales como Centro, Mediterráneo, Norte de España y el resto, para reducir los tamaños muestrales pequeños.

La actividad económica. Se trata de identificar el sector de la empresa a través de la Clasificación Nacional de Actividades Económicas (Código CNAE), se utiliza a nivel del dígito más agregado. Atendiendo a los trabajos de Jackson y Schuler (1995), que incluyen entre los factores contextuales influyentes en las prácticas de recursos humanos, las características del sector de actividad. Según Conway et al. (2008) se pueden categorizar de varios modos: servicios / industria. 
Tamaño. A tenor de los trabajos de Fields, Chan y Akhtar (2002, p. 265 y ss.), no parece haber consenso sobre la influencia de esta variable. Por un lado, Kortekaas (2007), halla un impacto de signo positivo del tamaño pequeño/medio en las conductas de los empleados y un impacto de signo negativo en el rendimiento operacional (bajas por enfermedad). Kok y Uhlaner (2001) establecen una relación entre el aumento del tamaño de las empresas y las prácticas de recursos humanos. Según Morgan (2014) las empresas pequeñas tienen varias ventajas como la flexibilidad de roles, relaciones, funciones y estilos de trabajo para los empleados, entre otros. En cambio, Ibrahim y Shah (2013) argumentan que las empresas pequeñas no disponen de los recursos necesarios para la puesta en marcha de prácticas de gestión de recursos humanos progresivas.

Con esta variable se busca saber el tamaño de las empresas que están en este ranking y si es susceptible de influir en la valoración total, debido a que cuanto más grande sea una empresa, más organizada ha de estar para ser competitiva. ¿Estar en el grupo de privilegio está reservado para las grandes corporaciones o el tamaño no es hecho relevante? En las estadísticas de carácter general, se agrupan entre las que superan los 250 empleados en España y las que están por debajo. En el análisis de regresión se usan los trabajadores de la empresa en España y en el mundo, porque no siempre existe correlación entre los trabajadores que tiene en un país y en el mundo.

Cotización. No hay investigaciones claras sobre los efectos de cotización en las políticas de recursos humanos (Conway et al, 2008). La selección de esta variable radica en que las empresas, antes de cotizar en el Mercado Secundario de Valores, han de pasar los requisitos y el control que exige la Comisión Nacional del Mercado de Valores, además de que los accionistas son más exigentes con el rendimiento de las empresas. Por ello, aparte de conocer si las empresas predominantes son las que cotizan en bolsa o no, ¿es ello un hecho relevante en la valoración obtenida por estas empresas?

\subsubsection{Variables usadas en el modelo de regresión}

Definen las mismas características que en el apartado anterior, pero se definen nuevas variables que agrupen las usadas en el análisis descriptivo para hacer el modelo de regresión global.

Tabla 3. Variables usadas en el modelo de regresión de las organizaciones más atractivas para el desempeño laboral en España.

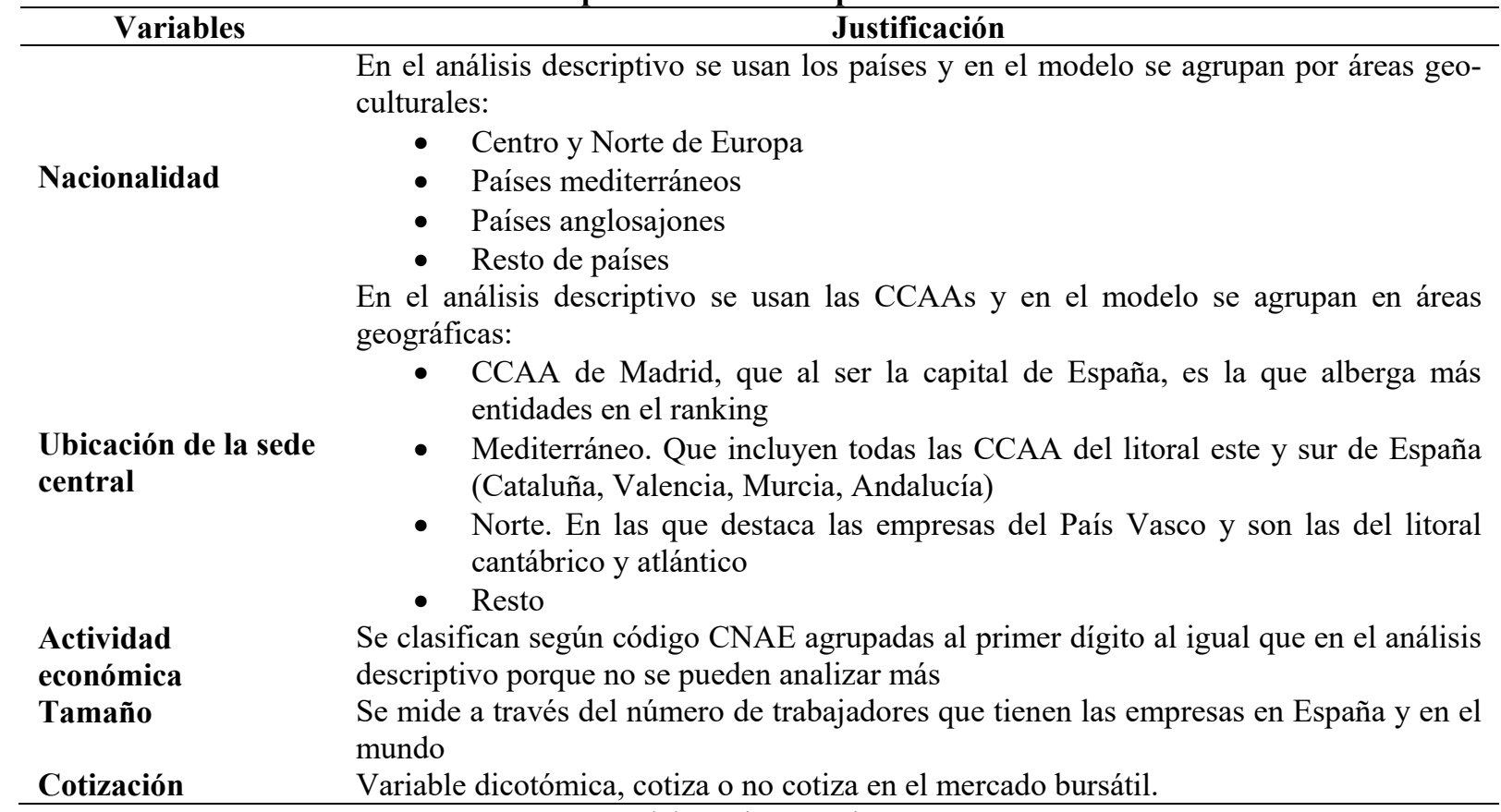

Fuente: Elaboración propia. 


\subsection{Muestra y Técnicas.}

Cada año, la $R A E$ publica una lista de las cien empresas que operan en España más valoradas para el desempeño laboral, de tal manera que para el período 2013-2016 tenemos una población de 400 observaciones. Este resultado basa su puntuación en un cuestionario enviado por expertos en materias en Recursos Humanos, con un centenar de preguntas que suman 1.000 puntos, distribuidas por variables según se aprecia en la Tabla 1, enviadas a más de 500 empresas que operan en España, que llevan más de cinco años en funcionamiento y deben tener una plantilla superior a los 100 empleados.

Una vez identificas las empresas del ranking publicadas por la $R A E$ para dicho período, se procede a identificar variables que permitan clasificar las empresas del ranking por países, CCAAs donde radica la sede en España, según actividades económica a través del CNAE, el tamaño de las empresas y si cotiza en bolsa, a través de estadísticos, medias ponderadas, porcentaje de ponderación, contraste de medias para diversos grupos con el test de Levene. Para el modelo de regresión se usan regresiones lineales múltiples mediante mínimos cuadrados ordinarios para cada uno de los cuatro años.

\section{Análisis descriptivo.}

Siguiendo el orden definido en la Tabla 2, se hace un análisis descriptivo que agrupa las empresas por nacionalidad, CCAA de la sede de empresa en España, actividad económica, tamaño y cotización.

\subsection{Nacionalidad de las empresas.}

En la Tabla 4 se puede apreciar el promedio de las empresas clasificadas según su nacionalidad. Así, observamos que proceden de hasta 17 países diferentes, mayoritariamente europeos, con presencia de organizaciones de EEUU (16,3\%), China (0,3\%), Corea del Sur $(1,0 \%)$ y Japón $(1,0 \%)$. Poco más de un tercio posee nacionalidad española $(38,8 \%)$, seguidas de las estadounidenses (16,3\%) y Reino Unido (10,0\%).

Analizando la valoración de las mismas, la media más alta corresponde a Corea del Sur, pero de este país sólo hay una empresa (LG), que aparece en todos los años dentro del ranking. Las empresas suizas, con 14 casos, obtienen una media de 799,9 puntos, destacando la farmacéutica Roche Farma y la aseguradora Zurich. Las empresas procedentes de EEUU y de España representan más de la mitad de la población, destacando entre las empresas americanas más valoradas Coca-Cola, que llegó a ocupar el primer puesto en el año 2013, Kimberly-Clarck, dedicada al sector sanitario, o las consultoras Pricewaterhouse o Deloitte. En España, las empresas más valoradas son las del sector financiero y seguros, tales como, BBVA o Banco Santander; o del sector energético, como Gas Natural e Iberdrola.

Aunque en el modelo de regresiones se analizará si es relevante o no la nacionalidad en la valoración promedio final, por los datos de la Tabla 4, no parece que existan causas vinculadas a los países que expliquen las diferencias de valor. Así, las empresas suizas que figuran entre las primeras posiciones, se podría pensar en que el elevado PIB/per cápita del país y su desarrollo económico pudiera ser una causa explicativa; en cambio los bajos valores obtenidos por las empresas suecas, incluso francesas o alemanas no apuntan en esa dirección. Tampoco se aprecia que los países anglosajones, exponentes de una amplia cultura empresarial y de mercado, obtengan unos resultados diferenciales.

Aunque no es objeto de este artículo analizar las diferencias de valores según el tipo de variable, pues se centra más en el valor promedio total y su posible dependencia de nuevos factores, se puede apreciar que los mayores valores promedios se obtienen en las dimensiones 
Talento $(176,7)$ y Formación $(175,2)$, si bien esto viene condicionado por los valores potenciales que pueden alcanzar, tal como se refleja en la Tabla 1. Por ello, al relacionar el valor promedio obtenido sobre su potencial, es la variable percepción de los empleados la que mejor se comporta, al obtener un valor 52,8 sobre un potencial de 60 puntos lo que supone un $88,1 \%$, seguida de la RSC con un $83,6 \%$, mientras que la peor valorada es la retribución al tener el valor más bajo respecto a su valor potencial $(71,4 \%)$.

Tabla 4. Organizaciones más atractivas para el desempeño (laboral) en España según "nacionalidad" ordenadas por promedio de valoración total.

\begin{tabular}{lccccccccc}
\hline Nacionalidad & Talento & Retribución & Ambiente & RSC & Formación & Empleados & Total & $\mathbf{N}^{\mathbf{0}}$ Empresas & $\mathbf{\%}$ \\
\hline Corea del Sur & 191,3 & 177,5 & 173,8 & 43,8 & 188,8 & 53,8 & 828,8 & 4,0 & $1,0 \%$ \\
Suiza & 183,2 & 165,0 & 169,6 & 44,3 & 183,4 & 54,5 & 799,9 & 14,0 & $3,5 \%$ \\
Irlanda & 175,0 & 152,5 & 168,8 & 48,8 & 187,5 & 56,5 & 789,0 & 4,0 & $1,0 \%$ \\
Japón & 175,0 & 165,0 & 161,3 & 48,8 & 173,3 & 54,0 & 777,3 & 4,0 & $1,0 \%$ \\
Finlandia & 192,5 & 162,5 & 160,0 & 35,0 & 165,0 & 60,0 & 775,0 & 2,0 & $0,5 \%$ \\
EEUU & 182,7 & 159,2 & 160,7 & 40,9 & 177,2 & 53,2 & 773,9 & 65,0 & $16,3 \%$ \\
España & 174,2 & 161,1 & 160,0 & 42,2 & 176,4 & 53,3 & 767,2 & 155,0 & $38,8 \%$ \\
Reino Unido & 182,9 & 158,6 & 156,9 & 41,9 & 171,9 & 53,5 & 765,6 & 40,0 & $10,0 \%$ \\
Italia & 167,8 & 186,1 & 146,1 & 42,2 & 165,6 & 54,0 & 761,8 & 9,0 & $2,3 \%$ \\
Países Bajos & 178,3 & 161,4 & 160,3 & 40,6 & 174,3 & 46,1 & 761,1 & 21,0 & $5,3 \%$ \\
Alemania & 175,0 & 164,2 & 156,1 & 41,4 & 168,8 & 54,9 & 760,3 & 28,0 & $7,0 \%$ \\
Francia & 177,5 & 159,1 & 150,4 & 41,6 & 178,4 & 52,1 & 759,1 & 34,0 & $8,5 \%$ \\
Portugal & 145,0 & 167,5 & 145,0 & 43,8 & 182,5 & 47,8 & 731,5 & 4,0 & $1,0 \%$ \\
Luxemburgo & 170,0 & 150,0 & 170,0 & 35,0 & 150,0 & 47,0 & 722,0 & 1,0 & $0,3 \%$ \\
Suecia & 169,6 & 139,6 & 152,3 & 38,3 & 170,0 & 51,4 & 721,2 & 12,0 & $3,0 \%$ \\
China & 175,0 & 150,0 & 125,0 & 30,0 & 130,0 & 40,0 & 650,0 & 1,0 & $0,3 \%$ \\
Dinamarca & 120,0 & 142,5 & 155,0 & 40,0 & 137,5 & 39,5 & 634,5 & 2,0 & $0,5 \%$ \\
Promedio & $\mathbf{1 7 6 , 7}$ & $\mathbf{1 6 0 , 7}$ & $\mathbf{1 5 8 , 5}$ & $\mathbf{4 1 , 8}$ & $\mathbf{1 7 5 , 2}$ & $\mathbf{5 2 , 8}$ & $\mathbf{7 6 5 , 8}$ & $\mathbf{4 0 0 , 0}$ & $\mathbf{1 0 0 , 0 \%}$ \\
general & & &
\end{tabular}

Fuente: Elaboración propia a partir de los datos publicados en Actualidad Económica ${ }^{\circ \mathrm{os}} 2.735$ (2013), 2.747 (2014), 2.758 (2015), 2.770 (2016).

\subsection{Ubicación de las empresas.}

En cuanto al análisis por Comunidades Autónomas en España, se puede apreciar en la Tabla 5 que las organizaciones más valoradas para el desempeño laboral se encuentran en 13 Comunidades Autónomas, siendo Madrid (76,8\%) con gran diferencia, la que mayor número de empresas acoge y con mayor número de empresas internacionales (17 países). La razón de esta concentración es por ser la capital de España y centro geográfico del país, que explica que la mayoría de empresas multinacionales extranjeras que operan en el país y muchas grandes empresas españolas se ubiquen en dicha comunidad. En segundo lugar, figura Cataluña (12,8\%), contando con empresas de siete nacionalidades diferentes. Llama la atención que el País Vasco tenga domiciliadas tan sólo 12 empresas, todas de nacionalidad española, al ser una de las comunidades autónomas con mayor desarrollo de España.

En cuanto al promedio del valor total de las empresas ordenadas por Comunidades Autónomas, se observa que las mayores puntuaciones se obtienen por organizaciones ubicadas en Cantabria (785 puntos), entre las que cabe destacar principalmente el Banco Santander. En segundo lugar, destacan las 51 empresas de Cataluña, que alcanzan la valoración media de 775,5 puntos, destacando empresas como la energética Gas Natural, que alcanzó la tercera posición en el año 2016; CaixaBank, del sector financiero, estando en novena posición en dicho año; o la 
farmacéutica Novartis. Las organizaciones pertenecientes al País Vasco, con un promedio del valor total de 771,6 puntos, se sitúan en tercer lugar, y se aprecia que las principales empresas proceden de dos sectores: del financiero, como el BBVA, que en el año 2015 alcanzó la primera posición, o del energético, como Iberdrola (posición 28 en 2016). La empresa Inditex, de origen gallego y una de las empresas con mayor valor del mundo, siendo su actividad principal la industria textil, coloca el promedio de las empresas gallegas en cuarto lugar.

La media general está justo por debajo de la valoración de las empresas de Madrid, que cuenta con una gran variedad de empresas, tanto por su nacionalidad como por la diversidad de sus actividades económicas, ocupando generalmente las principales posiciones, tales como CocaCola (primera en 2013), PWC (primera en 2014) o KPMG (primera en 2016).

Tabla 5. Organizaciones más valoradas para el desempeño laboral en España según "CCAA" ordenadas por promedio de valoración total.

\begin{tabular}{llllllllll}
\hline CCAA & Talento & Retribución & Ambiente & RSC & Formación & Empleados & Total & $\mathbf{N}^{\mathbf{0}}$ Empresas & \% \\
\hline Cantabria & 172,5 & 153,3 & 173,3 & 44,2 & 184,2 & 57,5 & 785,0 & 6 & $1,5 \%$ \\
Cataluña & 176,6 & 169,1 & 157,2 & 42,0 & 176,2 & 54,5 & 775,5 & 51 & $12,8 \%$ \\
País vasco & 175,8 & 161,7 & 160,2 & 41,3 & 180,0 & 52,7 & 771,6 & 12 & $3,0 \%$ \\
Galicia & 188,3 & 150,0 & 176,7 & 43,3 & 156,7 & 56,0 & 771,0 & 3 & $0,8 \%$ \\
Madrid & 177,6 & 160,6 & 158,7 & 41,8 & 175,3 & 52,5 & 766,6 & 307 & $76,8 \%$ \\
Islas Baleares & 180,0 & 160,8 & 146,0 & 37,5 & 181,7 & 51,2 & 757,2 & 6 & $1,5 \%$ \\
C. Valenciana & 153,3 & 150,0 & 140,0 & 41,7 & 178,3 & 55,3 & 718,7 & 3 & $0,8 \%$ \\
Andalucía & 161,3 & 146,3 & 157,5 & 40,0 & 166,3 & 46,8 & 718,0 & 4 & $1,0 \%$ \\
Aragón & 152,5 & 157,5 & 155,0 & 42,5 & 157,5 & 51,5 & 716,5 & 2 & $0,5 \%$ \\
Castilla y León & 165,5 & 137,5 & 165,0 & 47,5 & 142,5 & 53,0 & 711,0 & 2 & $0,5 \%$ \\
Islas Canarias & 185,0 & 115,0 & 155,0 & 45,0 & 160,0 & 50,0 & 710,0 & 1 & $0,3 \%$ \\
Asturias & 157,5 & 107,5 & 170,0 & 40,0 & 167,5 & 49,5 & 692,0 & 2 & $0,5 \%$ \\
Murcia & 145,0 & 105,0 & 130,0 & 40,0 & 155,0 & 60,0 & 635,0 & 1 & $0,3 \%$ \\
Promedio & & & & & & & & \\
general & $\mathbf{1 7 6 , 7}$ & $\mathbf{1 6 0 , 7}$ & $\mathbf{1 5 8 , 5}$ & $\mathbf{4 1 , 8}$ & $\mathbf{1 7 5 , 2}$ & $\mathbf{5 2 , 8}$ & $\mathbf{7 6 5 , 8}$ & $\mathbf{4 0 0}$ & $\mathbf{1 0 0 , 0} \%$ \\
\hline
\end{tabular}

Fuente: Elaboración propia a partir de los datos publicados en Actualidad Económica $\mathrm{n}^{\text {os }} 2.735$ (2013), 2.747 (2014), 2.758 (2015), 2.770 (2016).

\subsection{Actividad de la empresa.}

La Tabla 6 ofrece una visión de las empresas clasificadas según su actividad económica. El 24,3\% de las empresas se dedican al sector de las actividades financieras y de seguros; un 16,3\% al sector de actividades profesionales, científicas y técnicas; y un $14,3 \%$ al sector del comercio al por mayor y al por menor.

Las más valoradas, con pocas diferencias entre ellas, son las empresas del sector sanitario $(783,6)$, representadas por, entre otras, la empresa Kimberly-Clarck; el sector energético $(782,5)$, con empresas como Gas Natural-Fenosa, Endesa o Iberdrola; el de las actividades financieras (781,3), destacando BBVA y Banco Santander; y el sector de actividades profesionales, científicas y técnicas $(779,7)$, con las grandes consultoras, como Deloitte, KPMG. Las razones que explican estos resultados se pueden encontrar en que parece lógico pensar que el sector sanitario haga un especial énfasis en sus políticas de recursos humanos, de hecho, la percepción que tienen los empleados alcanza el 94,7\% del valor máximo. El sector energético requiere de personal técnico muy cualificado al igual que el sector financiero, que además con la crisis ha sufrido un proceso de concentración en el que aquellas entidades que no fueron competitivas fueron absorbidas, de tal manera que las que siguen operando han acreditado una eficiencia organizativa que no podrían alcanzar sin una política de excelencia en Recursos Humanos. 
Tabla 6. Organizaciones más valoradas para el desempeño laboral en España según "actividad económica" ordenadas por código CNAE.

\begin{tabular}{|c|c|c|c|c|c|c|c|c|c|c|}
\hline Código & CNAE & Talento & Retribución & Ambiente & RSC & Formación & Empleados & Total & $\begin{array}{c}\mathbf{N}^{\mathbf{o}} \\
\text { empresas }\end{array}$ & $\%$ \\
\hline A & $\begin{array}{l}\text { Agricultura, ganadería, } \\
\text { silvicultura y pesca }\end{array}$ & 170,0 & 155,0 & 155,0 & 50,0 & 135,0 & 50,0 & 715,0 & 1 & $0,3 \%$ \\
\hline $\mathrm{C}$ & $\begin{array}{l}\text { Industria manufacturera } \\
\text { Suministro de energía } \\
\text { eléctrica, gas, vapor y } \\
\text { aire acondicionado }\end{array}$ & 170,6 & 170,1 & 157,2 & 40,9 & 174,9 & 52,3 & 765,8 & 43 & $10,8 \%$ \\
\hline $\mathrm{F}$ & $\begin{array}{l}\text { Construcción } \\
\text { Comercio al por mayor y } \\
\text { al por menor; reparación } \\
\text { de vehículos de motor y }\end{array}$ & 179,3 & 152,1 & 136,7 & 39,3 & 180,7 & 55,0 & 743,1 & 7 & $1,8 \%$ \\
\hline G & $\begin{array}{l}\text { motocicletas } \\
\text { Transporte }\end{array}$ & 174,8 & 161,7 & 153,5 & 41,6 & 168,1 & 52,3 & 751,9 & 57 & $14,3 \%$ \\
\hline $\mathrm{H}$ & almacenamiento & 170,5 & 158,6 & 154,9 & 44,1 & 186,3 & 54,1 & 768,5 & 11 & $2,8 \%$ \\
\hline I & Hostelería & 176,1 & 135,6 & 152,8 & 43,9 & 181,7 & 56,4 & 746,4 & 9 & $2,3 \%$ \\
\hline $\mathrm{J}$ & $\begin{array}{l}\text { Edición } \\
\text { Actividades financieras }\end{array}$ & 175,3 & 157,7 & 159,8 & 41,5 & 169,0 & 52,2 & 755,5 & 49 & $12,3 \%$ \\
\hline $\mathrm{K}$ & $\begin{array}{l}\text { y de seguros } \\
\text { Actividades }\end{array}$ & 175,0 & 171,5 & 161,9 & 42,8 & 176,0 & 54,1 & 781,3 & 97 & $24,3 \%$ \\
\hline $\mathrm{L}$ & $\begin{array}{l}\text { inmobiliarias } \\
\text { Actividades } \\
\text { profesionales, científicas }\end{array}$ & 177,1 & 144,3 & 148,6 & 42,1 & 168,6 & 48,7 & 729,4 & 7 & $1,8 \%$ \\
\hline M & $\begin{array}{l}\text { y técnicas } \\
\text { Actividades } \\
\text { administrativas }\end{array}$ & 188,5 & 152,8 & 162,9 & 41,3 & 182,2 & 52,0 & 779,7 & 65 & $16,3 \%$ \\
\hline $\mathrm{N}$ & $\begin{array}{l}\text { servicios auxiliares } \\
\text { Administración pública }\end{array}$ & 179,8 & 143,0 & 154,0 & 39,6 & 170,9 & 50,1 & 737,3 & 23 & $5,8 \%$ \\
\hline $\mathrm{O}$ & $\begin{array}{l}\text { y defensa; seguridad } \\
\text { social obligatoria }\end{array}$ & 165,0 & 140,0 & 95,0 & 35,0 & 160,0 & 50,0 & 645,0 & 1 & $0,3 \%$ \\
\hline $\mathrm{P}$ & $\begin{array}{l}\text { Educación } \\
\text { Actividades sanitarias y }\end{array}$ & 127,5 & 130,0 & 157,5 & 40,0 & 172,5 & 44,5 & 672,0 & 2 & $0,5 \%$ \\
\hline Q & de servicios sociales & 170,7 & 164,3 & 162,9 & 45,7 & 183,3 & 56,7 & 783,6 & 7 & $1,8 \%$ \\
\hline & Total general & 176,7 & 160,7 & 158,5 & 41,8 & 175,2 & 52,8 & 765,8 & 400 & $100 \%$ \\
\hline
\end{tabular}

Fuente: Elaboración propia a partir de los datos publicados en Actualidad Económica $\mathrm{n}^{\text {os }} 2.735$ (2013), 2.747 (2014), 2.758 (2015), 2.770 (2016).

\subsection{Tamaño de las empresas.}

En este apartado se analizará la variable tamaño, medida como el número de trabajadores que poseen las empresas más atractivas para el desarrollo profesional en España, clasificándolas en dos grandes grupos, las de menos y más de 250 trabajadores (Tabla 7), siendo la media de trabajadores de esta población de empresas de 5.116,4.

Se aprecia una mejor puntuación en las empresas de mayor dimensión, que representan el $87,3 \%$ del total, en todos los ítems, exceptuando la gestión del talento. Por otro lado, se puede observar que las empresas muy grandes, tales como Coca-Cola, Roche Farma u Orange, ocupan las primeras posiciones, con alguna excepción, como la empresa anglosajona BAT, dedicada a la industria del tabaco, que en España tiene menos de 250 trabajadores y que ocupa las primeras posiciones. La causa por la que parece que el tamaño es un factor relevante podría ser que una organización grande ha tenido necesariamente que crecer, y para esto se necesita una eficiencia organizativa y una buena gestión de recursos humanos. 
Tabla 7. Organizaciones más valoradas para el desempeño laboral en España según "tamaño" ordenadas por promedio de valoración total.

\begin{tabular}{|c|c|c|c|c|c|c|c|c|c|}
\hline Empleados & Talento & Retribución & Ambiente & RSC & Formación & Empleados & Total & $\begin{array}{c}\mathbf{N}^{\mathbf{o}} \\
\text { empresas }\end{array}$ & $\%$ \\
\hline$>250$ & 176,1 & 161,5 & 159,4 & 42,1 & 176,1 & 53,1 & 768,3 & 349,0 & $87,3 \%$ \\
\hline Total general & 176,7 & 160,7 & 158,5 & 41,8 & 175,2 & 52,8 & 765,8 & 400,0 & $100,0 \%$ \\
\hline
\end{tabular}

Fuente: Elaboración propia a partir de los datos publicados en Actualidad Económica $\mathrm{n}^{\text {os }} 2.735$ (2013), 2.747 (2014), 2.758 (2015), 2.770 (2016).

En la Tabla 8, se aprecia que se trata de un contraste de diferencia de medias en el que se concluye que las varianzas son distintas y las medias son distintas ( $\mathrm{p}$-valor bilateral de 0,23 ); por lo que la valoración media de las grandes empresas es superior significativamente con un p-valor (unilateral) de 0,046.

Tabla 8. Estadísticos y prueba de muestras independientes de la Valoración Total clasificadas por tamaños de empresas.

\begin{tabular}{|c|c|c|c|c|c|c|c|}
\hline \multicolumn{2}{|c|}{ Medidas/Tamaño } & $\mathbf{N}$ & Media & F. & Sig. & $\begin{array}{l}\text { Prueba de } \\
\text { Levene }\end{array}$ & $\begin{array}{c}\text { Sig. } \\
\text { (bilateral) }\end{array}$ \\
\hline \multirow{2}{*}{ Total } & \multirow{2}{*}{$\begin{array}{l}\qquad \begin{array}{c}=<250 \\
\text { profesionales }\end{array} \\
\quad>250 \\
\text { profesionales }\end{array}$} & 51 & 749,0000 & 4,073 & \multirow[t]{2}{*}{,044 } & $\begin{array}{c}\text { No se } \\
\text { asumen }\end{array}$ & \multirow{2}{*}{,023 } \\
\hline & & 349 & 768,3009 & & & $\begin{array}{l}\text { varianzas } \\
\text { iguales }\end{array}$ & \\
\hline
\end{tabular}

Fuente: Elaboración propia a partir de los datos publicados en Actualidad Económica n $^{\text {os }} 2.735$ (2013), 2.747 (2014), 2.758 (2015), 2.770 (2016).

\subsection{Cotización en el mercado bursátil.}

La Tabla 9 muestra las principales empresas más atractivas para trabajar en España clasificadas según si cotizan o no en el mercado bursátil, resultando que más de la mitad de las empresas $(67,3 \%)$ están presentes en el mercado de valores, a la vez que son las que obtienen una mejor valoración en todos los ítems y ocupan, por lo tanto, las primeras posiciones. Esto pudiera explicarse por el hecho de que una empresa que cotiza en el mercado bursátil está más exigida por la Comisión Nacional de Valores, además de que los accionistas están muy atentos a la evolución de la empresa, que ha de buscar permanentemente la eficiencia en todos los ámbitos de la empresa.

Tabla 9. Organizaciones más valoradas para el desempeño laboral en España según "cotice en el mercado bursátil" ordenadas por promedio de valoración total.

\begin{tabular}{|c|c|c|c|c|c|c|c|c|c|}
\hline $\begin{array}{c}\text { Cotización } \\
\text { mercado de } \\
\text { valores }\end{array}$ & Talento & Retribución & Ambiente & RSC & Formación & Empleados & Total & $\begin{array}{c}\mathbf{N}^{0} \\
\text { empresas }\end{array}$ & $\%$ \\
\hline No cotiza & 174,1 & 154,0 & 156,2 & 40,9 & 171,6 & 51,9 & 748,8 & 131,0 & $32,8 \%$ \\
\hline Cotiza & 178,0 & 163,9 & 159,7 & 42,3 & 177,0 & 53,3 & 774,2 & 269,0 & $67,3 \%$ \\
\hline $\begin{array}{c}\text { Total } \\
\text { general }\end{array}$ & 176,7 & 160,7 & 158,5 & 41,8 & 175,2 & 52,8 & 765,8 & 400,0 & $100,0 \%$ \\
\hline
\end{tabular}

Fuente: Elaboración propia a partir de los datos publicados en Actualidad Económica n ${ }^{\text {os }} 2.735$ (2013), 2.747 (2014), 2.758 (2015), 2.770 (2016). 
En la Tabla 10, se puede apreciar que se trata de un contraste de diferencia de medias en el que se concluye que las varianzas son distintas y las medias son distintas (p-valor bilateral de $0,000)$, por lo que resulta que los valores medios de las empresas que cotizan son significativamente superiores a las que no cotizan.

Tabla 10. Estadísticos y prueba de muestras independientes de la Valoración Total según cotización de las empresas.

\begin{tabular}{cccccccc}
\hline \multicolumn{2}{c}{$\begin{array}{c}\text { Medidas/Cotización } \\
\text { Mercado Bursatil }\end{array}$} & N & Media & F. & Sig. & $\begin{array}{c}\text { Prueba de } \\
\text { Levene }\end{array}$ & $\begin{array}{c}\text { Sig. } \\
\text { (bilateral) }\end{array}$ \\
\hline \multirow{2}{*}{ Total } & No cotiza & 131 & 748,7557 &, 935 &, 334 & $\begin{array}{c}\text { Se asumen } \\
\text { varianzas } \\
\text { iguales }\end{array}$ &, 000 \\
& Sí cotiza & 269 & 774,1599 & & & & \\
\hline
\end{tabular}

Fuente: Elaboración propia a partir de los datos publicados en Actualidad Económica n ${ }^{0 \mathrm{~s}} 2.735$ (2013), 2.747 (2014), 2.758 (2015), 2.770 (2016).

\section{Modelo de regresión.}

Tras mostrar las diferencias entre las valoraciones de las empresas en análisis descriptivos, se estimará en qué medida modifican el atractivo de las empresas para trabajar en ellas, que se mide con la valoración total, las diferencias en los valores de las variables independientes, manteniendo constantes las demás variables. Para ello, se estima un modelo de regresión lineal múltiple mediante mínimos cuadrados ordinarios para cada uno de los cuatro años. En cada uno de los modelos, la variable dependiente es la valoración total de la empresa. Aun cuando las variables independientes, ya están explicadas anteriormente, se entiende que clarifica relacionarlas en este apartado, que son las que se listan seguidamente:

1. El área geográfica española a la que pertenece la Comunidad Autónoma sede de la empresa, que es una variable categórica con los siguientes valores: Mediterráneo, Norte y resto de áreas, siendo el área de referencia la Comunidad de Madrid.

2. El área geo-cultural del país de nacionalidad de la empresa, variable también categórica con los siguientes valores: Centro y Norte de Europa, Países mediterráneos europeos y resto de países, siendo el grupo de referencia el de los países anglosajones.

3. La nacionalidad española de la empresa, variable dicotómica, siendo el grupo de referencia las empresas de nacionalidad no española.

4. El tamaño de la empresa, variable dicotómica con las categorías grande y pequeña, siendo el grupo de referencia el de las empresas pequeñas.

5. El número de trabajadores de la empresa en todo el mundo.

6. El número de trabajadores de la empresa en España.

7. La cotización en Bolsa, variable dicotómica, siendo el grupo de referencia el de las empresas que no cotizan en Bolsa.

8. El sector de actividad económica de la empresa según la categorización de la CNAE, siendo el grupo de referencia el sector Agricultura, Ganadería, Silvicultura y Pesca.

En todos los modelos, el número de observaciones es idéntico: las 100 empresas listadas por Actualidad Económica en el año en cuestión.

La Tabla 11 muestra los resultados de los cuatro modelos de regresión. Ninguno de los modelos es estadísticamente significativo (para todos ellos, $p(F)>.1$ ). A pesar de que la falta de significación estadística de los modelos, es posible apuntar algunas observaciones relevantes para futuras y más profundas investigaciones.

La única variable independiente que alcanza significación estadística (al nivel del .01) en al menos dos modelos (2015 y 2016) es la cotización en Bolsa. En ambos casos, las empresas que 
cotizan en Bolsa tienen una mayor valoración, aumentando el intercepto en 23.44 y 58.98 puntos, respectivamente.

Tabla 11. Análisis global de las organizaciones más atractivas para el desempeño (laboral) en

\begin{tabular}{|c|c|c|c|c|}
\hline \multirow[t]{2}{*}{$x^{2}$} & \multicolumn{2}{|l|}{ España. } & \\
\hline & 2013 & 2014 & 2015 & 2016 \\
\hline \multirow[t]{2}{*}{ Intercepto } & $662.16^{* * *}$ & $748.06 * * *$ & $759.21 * * *$ & $753.73 * * *$ \\
\hline & $(41.88)$ & (76.10) & $(35.44)$ & $(63.77)$ \\
\hline \multirow[t]{2}{*}{ CCAA Mediterráneo } & -4.80 & -16.78 & -5.83 & 3.35 \\
\hline & $(17.92)$ & $(17.05)$ & (13.98) & $(27.21)$ \\
\hline \multirow[t]{2}{*}{ CCAA Norte } & -31.50 & -.93 & 32.16 & -54.38 \\
\hline & $(25.75)$ & $(32.63)$ & $(28.47)$ & $(42.08)$ \\
\hline \multirow[t]{2}{*}{ CCAA resto } & & -35.48 & -3.16 & -48.92 \\
\hline & & $(41.43)$ & $(27.84)$ & $(55.93)$ \\
\hline \multirow[t]{2}{*}{ Países Centro y Norte Europa } & 22.15 & $77.98 *$ & 56.85 & 12.05 \\
\hline & $(35.75)$ & $(42.77)$ & $(35.42)$ & $(68.46)$ \\
\hline \multirow[t]{2}{*}{ Países Mediterráneos Europa } & 4.15 & 4.83 & -12.42 & -19.37 \\
\hline & $(17.61)$ & $(17.60)$ & $(14.47)$ & $(28.79)$ \\
\hline \multirow[t]{2}{*}{ Resto de países } & -20.04 & -30.53 & -.35 & 4.58 \\
\hline & $(20.97)$ & $(20.89)$ & $(16.84)$ & $(34.24)$ \\
\hline \multirow[t]{2}{*}{ Nacionalidad española } & 25.86 & 25.12 & 8.47 & 7.87 \\
\hline & $(22.82)$ & $(22.10)$ & $(17.95)$ & $(31.35)$ \\
\hline \multirow[t]{2}{*}{ Tamaño } & $40.77 * *$ & 2.82 & -2.61 & -8.31 \\
\hline & $(18.68)$ & $(19.51)$ & $(16.18)$ & $(30.04)$ \\
\hline \multirow[t]{2}{*}{ Número de trabajadores (Mundo) } & -0.00 & 0.00 & 0.00 & 0.00 \\
\hline & $(0.00)$ & $(0.00)$ & $(0.00)$ & $(0.00)$ \\
\hline \multirow[t]{2}{*}{ Número de trabajadores (España) } & 0.00 & 0.00 & 0.00 & -0.00 \\
\hline & $(0.00)$ & $(0.00)$ & $(0.00)$ & $(0.00)$ \\
\hline \multirow[t]{2}{*}{ Cotización en bolsa } & 23.94 & 8.02 & $23.44^{*}$ & $58.98 * * *$ \\
\hline & $(16.00)$ & $(15.53)$ & (12.87) & $(21.51)$ \\
\hline \multirow[t]{2}{*}{$C N A E$ Industria Manufacturera } & & -3.72 & & \\
\hline & & $(68.04)$ & & \\
\hline \multirow[t]{2}{*}{$\begin{array}{l}C N A E \text { Suministro de Energía } \\
\text { Eléctrica, gas, vapor. }\end{array}$} & -21.09 & 18.12 & 9.77 & 26.81 \\
\hline & (31.22) & (74.09) & $(26.32)$ & $(56.24)$ \\
\hline \multirow[t]{2}{*}{$C N A E$ Construcción } & 18.29 & 54.21 & 16.17 & $-112.13^{*}$ \\
\hline & $(61.38)$ & $(89.34)$ & $(36.24)$ & $(60.49)$ \\
\hline \multirow[t]{2}{*}{$\begin{array}{l}C N A E \text { Comercio al por mayor y al por } \\
\text { menor }\end{array}$} & .30 & -18.68 & -6.25 & -61.08 \\
\hline & $(23.29)$ & $(71.04)$ & $(18.76)$ & $(40.39)$ \\
\hline \multirow[t]{2}{*}{$C N A E$ Transporte y almacenamiento } & 7.49 & -6.69 & -27.92 & 34.30 \\
\hline & (44.87) & (73.91) & $(31.47)$ & $(70.22)$ \\
\hline$C N A E$ Hostelería & -39.54 & -24.01 & -26.82 & -86.95 \\
\hline
\end{tabular}




\begin{tabular}{|c|c|c|c|c|}
\hline & 2013 & 2014 & 2015 & 2016 \\
\hline & $(47.91)$ & $(75.79)$ & $(31.93)$ & $(100.02)$ \\
\hline \multirow[t]{2}{*}{$C N A E$ Edición } & -10.13 & 4.73 & -14.26 & $-86.48^{*}$ \\
\hline & $(23.40)$ & $(70.09)$ & $(21.65)$ & $(44.54)$ \\
\hline \multirow[t]{2}{*}{$\begin{array}{l}C N A E \text { Actividades financieras y de } \\
\text { seguros }\end{array}$} & 21.55 & 25.63 & 23.95 & -17.24 \\
\hline & $(21.10)$ & $(69.75)$ & (17.71) & $(36.32)$ \\
\hline \multirow[t]{2}{*}{$C N A E$ Actividades inmobiliarias } & -12.15 & -7.20 & -32.55 & -76.46 \\
\hline & $(61.24)$ & $(78.25)$ & $(36.01)$ & $(72.26)$ \\
\hline \multirow[t]{2}{*}{$\begin{array}{l}C N A E \text { Actividades profesionales, } \\
\text { científicas }\end{array}$} & 26.87 & 17.18 & $33.41^{*}$ & 13.75 \\
\hline & $(22.53)$ & $(67.54)$ & $(19.37)$ & $(38.06)$ \\
\hline \multirow[t]{2}{*}{$\begin{array}{l}C N A E \text { Actividades administrativas y } \\
\text { servicios auxiliares }\end{array}$} & -30.96 & -14.17 & -3.06 & -55.19 \\
\hline & $(33.54)$ & $(71.97)$ & $(25.80)$ & $(45.64)$ \\
\hline \multirow[t]{2}{*}{ CNAE Administración pública } & & & & -108.31 \\
\hline & & & & $(95.20)$ \\
\hline \multirow[t]{2}{*}{ CNAE Educación } & & -41.77 & & -119.60 \\
\hline & & $(89.13)$ & & $(92.13)$ \\
\hline \multirow[t]{2}{*}{$\begin{array}{l}C N A E 1 \text { Actividades sanitarias y de } \\
\text { servicios sociales }\end{array}$} & $104.30^{*}$ & -8.53 & 15.45 & -3.19 \\
\hline & $(59.22)$ & $(75.20)$ & $(35.83)$ & $(92.39)$ \\
\hline Observaciones & 100 & 100 & 100 & 100 \\
\hline$R^{2}$ & .25 & .22 & .25 & .31 \\
\hline$R^{2}$ Ajustado & .05 & -.03 & .03 & .09 \\
\hline Error típico residual & $56.07(\mathrm{df}=78)$ & $54.23(\mathrm{df}=75)$ & $45.30(\mathrm{df}=77)$ & $85.27(\mathrm{df}=75)$ \\
\hline$F$ & $\begin{array}{l}1.26(\mathrm{df}=21 \\
78)\end{array}$ & $\begin{array}{l}.89(\mathrm{df}=24 \\
75)\end{array}$ & $\begin{array}{l}1.16(\mathrm{df}=22 ; \\
77)\end{array}$ & $\begin{array}{l}1.43(\mathrm{df}=24 ; \\
75)\end{array}$ \\
\hline Notas. & $* * * p<.01 ; * * p$ & $<.05 ; * p<.1$ & & \\
\hline
\end{tabular}

Fuente: Elaboración propia a partir de los datos publicados en Actualidad Económica n ${ }^{\text {os }} 2.735$ (2013), 2.747 (2014), 2.758 (2015), 2.770 (2016).

El tamaño de la empresa también tiene significación estadística, al menos en 2013 ( $p<$ $.05)$ : las empresas grandes parecen ser más valoradas (40.77 puntos) que las pequeñas.

El área geo-cultural de la nación de la empresa tiene significación estadística (la prueba Anova para modelos anidados, en la que el modelo completo es el estimado en este trabajo y el modelo reducido es el mismo modelo con la exclusión de las tres variables dummy que representan el área geo-cultural, arroja una $F=2.3, p(F)<.1)$. El área norte y centro-europea $(p<.1)$ parece aumentar la valoración de las empresas respecto al área anglosajona en 2014 en casi 78 puntos.

El sector de actividad económica parece carecer de significación estadística alguna. Para todos los años, la prueba Anova para modelos anidados (siendo el modelo completo el estimado en este trabajo para cada año, y el modelo reducido el mismo modelo sin las variables dummy de actividad económica según la clasificación de la CNAE) arroja una $p(F)$ muy superior a .1. 
Si se atiende al signo de los coeficientes, la pertenencia de las empresas a un área geográfica española distinta de Madrid parece reducir su atractivo para trabajar en ellas. Por otra parte, si la pertenencia de las empresas al área geo-cultural norte y centro-europea parece aumentar su puntuación respecto a las empresas del área anglosajona, la pertenencia al área mediterránea y, sobre todo, a otros países parece contribuir a reducir el atractivo de las empresas.

El tamaño de las empresas, clasificando a las empresas en grandes y pequeñas, aumenta su atractivo en 2013 y 2014, pero lo reduce en los dos años sucesivos. La variable cotización en Bolsa tiene sistemáticamente el mismo signo positivo: cotizar en Bolsa aumenta el atractivo de las empresas.

Analizando las causas por las que la nacionalidad no indica ser un factor relevante pudiera ser que, al tratarse de las cien empresas más valoradas para el desarrollo profesional, no existan diferencias significativas por su condición de ser las mejores, y de pertenecer a un ranking de empresas selectas, además de que estas empresas, que son mayoritariamente multinacionales, proceden de los países más desarrollados del mundo, como se puede apreciar en la Tabla 4. Lo mismo pasa al hacer el análisis por áreas geográficas y culturales, pues no hay ninguna razón para pensar que el valor obtenido por las empresas del Mediterráneo, en el que se ubica Cataluña, tengan una diferencia relevante respecto a las asentadas en el centro, o a las del norte, con el País Vasco o Cantabria. Dado que las empresas del presente ranking están presentes en 15 sectores económicos, al hacer al análisis de regresión por años y con un total de 100 empresas en total por año, hace que el propio tamaño muestral se pudiera no mostrar con relevancia estadística, a pesar de que las empresas de los sectores energético, sanitario y financieros obtengan los mayores valores. Que las empresas según su tamaño y cotización bursátil manifiesten valores más relevantes, obedece a las exigencias de cotizar en el Mercado Nacional de Valores, y de que generalmente una organización que crece ha de tener modelos de eficiencia empresarial.

\section{Conclusiones.}

Un tercio de las cien empresas más valoradas para trabajar son españolas, seguidas de las estadounidenses (16,3\%) y del Reino Unido (10,0\%). Al clasificarlas según su valoración, son las empresas de fuera de la Unión Europea las más valoradas: las de Corea del Sur, Suiza y Japón. Es llamativo que las empresas de los países nórdicos, como Dinamarca o Suecia, sean las menos valoradas, o que las alemanas, francesas, italianas y de los Países Bajos estén por debajo de la media. La no relevancia de este factor, en la línea de las conclusiones de Ibrahim y Shah (2013) y en sentido contrario a los trabajos de Ferner (1997), Liu (2004) y Guthrie et al. (2008), se puede explicar por el hecho de que proceden de países desarrollados y nos estamos centrando en las cien empresas más atractivas para el desarrollo laboral, lo que hace que, aunque el valor varíe según el país, no resulten diferencias relevantes, ni aún agrupándolas por áreas geográficas. En todo caso es un hallazgo identificar de qué países son las empresas más excelentes para trabajar en España, en qué cuantía, con qué valores, y que no existen diferencias relevantes desde el punto de vista estadístico. Por tanto, un profesional que trabaje en una empresa española, no tiene menos relevancia que otra empresa de otro país, lo que lleva a la conclusión de que las empresas españolas de esta muestra no tienen un menor nivel de excelencia en los recursos humanos que las extranjeras.

En cuatro de las diecisiete regiones españolas no hay ninguna empresa en el ranking para el período 2013-16. La Comunidad de Madrid alberga el 76,8\% de las mismas, seguida de las catalanas, con el 12,8\%. Destacan por su valoración promedio las situadas en Cantabria, Cataluña, País Vasco y Galicia. Al igual que en los países, y en contra de los resultados de Amossé et al., 2016; Conway et al., 2008; Fields, Chan y Akhtar, 2002; Grimshaw y Rubery, 2007; Paawee y Boselie, 2007; la no relevancia a nivel valorativo se pudiera explicar en que, al tratarse de las empresas más excelentes, no existen razones de peso por el hecho de que se ubiquen en Madrid, en Cataluña o País Vasco, y ni siguiera al agrupar por zonas geo-culturales. Por tanto, la 
contribución es además de identificar en qué zona de España se ubican sus sedes centrales, las empresas ubicadas en Madrid no tienen valores más relevantes que las de otras zonas de España.

Al vincular las empresas a la actividad principal de las mismas, las del sector financiero y de seguros son las más numerosas y las segundas más valoradas. Las dedicadas a las actividades profesionales, científicas y técnicas ocupan la segunda posición en número y la tercera en valoración media, siendo las más valoradas las del sector sanitario, energético y financieras. No obstante, la presencia de empresas de quince sectores en una muestra de 100 empresas, hacen que los tamaños muestrales para cada año salgan muy poco relevantes y no se puede concluir que son relevantes las empresas según la actividad a la que se dedica.

Salvo en la variable independiente Gestión del Talento, obtienen mayores valoraciones medias las empresas con más de 250 empleados, que suponen el $87,3 \%$ del total. Tener un mayor tamaño implica crecer y esto obliga a modelos de excelencia en las políticas de recursos humanos, siendo una contribución el identificar el tamaño como un factor con relevancia estadística, en línea a las conclusiones de Kok y Uhlaner (2001) que apreciaron una relación entre el aumento del tamaño de las empresas y las prácticas de recursos humanos.

Al clasificar las empresas por cotización en el mercado bursátil, las que lo hacen representan el 67,3\% y superan a las que no lo hacen en todas las variables analizadas. Es el factor que mayor relevancia muestra, motivado porque las empresas que cotizan en bolsa, tienen mayores niveles de exigencia por parte del mercado y de las instituciones, lo que les obliga a desarrollar una mayor eficiencia organizativa y en su política de recursos humanos. Ésta es una aportación novedosa ya que no había investigaciones claras sobre los efectos de cotización en las políticas de recursos humanos (Conway et al., 2008, p. 638).

El modelo de regresión para los cuatro años no muestra una capacidad explicativa de la varianza de la variable dependiente. Futuras investigaciones deberían centrarse en especificar el modelo incorporando nuevas variables (como pudieran ser la cultura organizativa o el estilo de liderazgo).

\section{Referencias}

Alles, M. (2006). Desarrollo del talento humano basado en competencias. Buenos Aires: Ediciones Granica, S.A.

Amossé, T., Bryson, A., Forth, J., \& Petit, H. (2016). Managing and working in Britain and France: An introduction. En: T. Amossé, A. Bryson, J. Forth \& H. Petit (Eds.), Comparative workplace employment relations. An analysis of practice in Britain and France (pp. 1-26). Londres: Palgrave MacMillan. DOI: 10.1057/978-1-137-57419-0_1.

Arikan, E., Kantur, C., Maden, C., \& Telci, E.E. (2016). Investigating the mediating role of corporate reputation on the relationship between corporate social responsibility and multiple stakeholder outcomes. Quality \& Quantity, 50, 129-149.

Armstrong, M. (2006). A handbook of human resource management practice. London: Kogan Page.

Ashton, C., \& Morton, L. (2005). Managing talent for competitive advantage. Strategic HR Review, 4(5), 28-31. DOI: 10.1108/14754390580000819.

Ballou, B., Godwin, N.H., \& Shortridge, R.T. (2003). Firm value and employee attitudes on workplace quality. Accounting Horizons, 17(4), 329-341. DOI: 10.2308/acch.2003.17.4.329. 
Betcherman, G., Leckie, N., \& McMullen, K. (1997). Developing Skills in the Canadian Workplace: The Results of the EKOS Workplace Training Survey. Canadian Policy Research Networks, Otawa. Recuperado de https://www.voced.edu.au/content/ngv\%3A31933

Bešlić, I., \& Bešlić, D. (2008). Zadovoljstvo poslom. Škola biznisa, 3, 154-162.

Bethke-Langenegger, P., Mahler, P., \& Staffelbach, B. (2011). Effectiveness of talent management strategies. European Journal of International Management, 5(5), 524-539. DOI: 10.1504/EJIM.2011.042177.

Bonache, J. (1996). El papel de la política de recursos humanos en la internacionalización de la empresa. Economía Industrial, 307, 37-48.

Brown, M. (2001). Unequal pay, unequal responses? Pay referents and their implications for pay level satisfaction. Journal of Management Studies, 38(6), 879-896. DOI: 10.1111/1467-6486.00263.

Capraro, A. J., \& Srivastava, R.K. (1997). Has the influence of financial performance on reputation measures been overstated? Corporate Reputation Review, 1, 86-92. DOI: 10.1057/palgrave.crr.1540023.

Clark, A., Kristensen, N., \& Westergard-Nielsen, N. (2007). Job satisfaction and co-worker wages: Status or signal? The Economic Journal, 119(536), 430-447. DOI: 10.1111/j.14680297.2008.02236.x.

Conway, N., Deakin, S., Komzelmann, S., Petit, H., Rebérioux, A., \& Wilkinson, F. (2008). The influence of stock market listing on human resource management: Evidence for France and Britain. British Journal of Industrial Relations, 46(4), 632-673. DOI: 10.1111/j.14678543.2008.00698.x.

Davies, G., Chun, R., Vinhas da Silva, R., \& Roper, S. (2003). Corporate reputation and competitiveness. London and New York: Routledge.

DiRomualdo, T., Joyce, S., \& Bression, N. (2009). Key Findings from Hackett's Performance Study on Talent Management Maturity. Palo Alto, CA: Hackett Group.

Elsbach, K.D., \& Glynn, M.A. (1996). Believing your own 'PR': Embedding Identification in Strategic Reputation. Advances in Strategic Management, 13, 65-90.

Fernández-Gámez, M.A., Gil-Corral, A.M., \& Galán-Valdivieso, F. (2016). Corporate reputation and market value: Evidence with generalized regression neural networks. Expert Systems with Applications, 46, 69-76. DOI: 10.1016/j.eswa.2015.10.028.

Ferner, A. (1997). Country of origin effects and HRM in multinational companies. Human Resource Management Journal, 7(1), 19-37. DOI: 10.1111/j.1748-8583.1997.tb00271.x.

Fields, D., Chan, A., \& Akhtar, S. (2002). Organizational context and human resource management strategy: A structural equation analysis of Hong Kong firms. International Journal of Human Resource Management, 11(2), 264-277. DOI: $10.1080 / 095851900339864$.

Finegold, D., \& Sosckice, D. (1988). The Failure of British Training: Analysis and Prescription. 
Oxford Review of Economic Policy, 4, 21-53. Recuperado de https://www.jstor.org/stable/23606208?seq=1

Fombrun, C. J., \& Shanley, M. (1990). What's in a name? Reputation building and corporate strategy. Academy of Management Journal, 33(2), 233-258. DOI: 10.2307/256324.

Friedman, R. (2014). The best place to work. The art and science of creating an extraordinary workplace. Nueva York: Penguin.

Fulmer, I.S., Gerhart, B., \& Scott, K.S. (2003). Are the 100 Best better? An empirical investigation of the relationship between being a "Great Place to Work" and firm performance. Personnel Psychology, 56(4), 965-993. DOI: 10.1111/j.17446570.2003.tb00246.x.

Gandossy, R., \& Kao, T. (2004). Talent wars: out of mind, out of practice. Human Resource Planning, 27(4), 15-19.

Grimshaw, D., \& Rubery, J. (2007). Economics and HRM. En P. Boxall, J. Purcell \& P. Wright (Eds.), The Oxford Handbook of Human Resource Management (pp. 68-87). Nueva York: Oxford UniverSity Press.

Guest, D.E. (2007). HRM and the worker: Towards a new psychological contract? En P. Boxall, J. Purcell y P. Wright (Eds.), The Oxford Handbook of Human Resource Management (pp. 128-146). Oxford: Oxford Univerity Press.

Guinot, J., Chiva, R., \& Mallén, F. (2015). Altruismo y capacidad de aprendizaje organizativo: Un estudio en las empresas mejor valoradas por los trabajadores en España. Universia Business Review, 45, 92-109.

Guthrie, J.P., Liu, W., Flood, P.C., \& MacCurtain, S. (2008). High performance work systems, workforce productivity, and innovation: A comparison of MNCs and indigenous firms (WP 04-08). Recuperado de doras.dcu.ie/2421/1/wp0408.pdf

Hall, R. (1992). The strategic analysis of intangible resources. Strategic Management Journal, 13, 145-158. DOI: 10.1002/smj.4250130205.

Helm, S. (2007). One Reputation or Many? Comparing Stakeholders' Perceptions of Corporate Reputation. Corporate Communications: An International Journal, 12(3), 238-254.

Hinkin, T.R., \& Tracey, J.B. (2010). What makes it so great? An analysis of human resources practices among Fortune's Best Companies to Work for. Cornell Hospitality Quarterly, 51(2), 158-170. DOI: 10.1177/1938965510362487.

Hosie, P., Jayashree, P., Tchantchane, A., \& Ban, L. (2013). The effect of autonomy, training opportunities, age and salaries on job satisfaction in the South East Asian retail petroleum industry. The International Journal of Human Resource Management, 24(21), 3980-4007. DOI: $10.1080 / 09585192.2013 .829517$.

Ibrahim, H.I., \& Shah, K.A.M. (2013). Effects of organizational characteristics factors on the implementation of strategic human resource practices: Evidence from Malaysian manufacturing firms. ECONOMIA. Seria MANAGEMENT, 16(1), 5-24. 
Jackson, S., \& Schuler, R.S. (1995). Understanding human resource management in the context of organizations and their environments. Annual Review of Psychology, 46, 237-264. DOI: 10.1146/annurev.ps.46.020195.001321.

Joo, B. K., \& McLean, G.N. (2006). Best employer studies: A conceptual model from a literature review and a case study. Human Resource Development Review, 5(2), 228-257. DOI: $10.1177 / 1534484306287515$.

Joyce, K.E. (2003). Lessons for employers from Fortune's "100 best". Business Horizons, 46(2), 77-84. DOI: $10.1016 / \mathrm{S} 0007-6813(03) 00013-2$

Kabak, K.E., Şen, A., Gocer, K., Küçüksöylemez, S., \& Tuncer, G. (2014). Strategies for employee job satisfaction: A case of service sector. Procedia - Social and Behavioral Sciences, 150, 1167-1176. DOI: 10.1016/j.sbspro.2014.09.132.

Khota, S., Rajgopal, S., \& Rindova, V.P. (2001). Reputation building and performance: an empirical analysis of the top-50 pure internet firms. European Management Journal, 19(6), 571-586. DOI: 10.1016/S0263-2373(01)00083-4.

Kok, J., \& Uhlaner, L.M. (2001). Organization context and human resource management in the small firm. Small Business Economics, 17(4), 273-291. DOI: 10.1023/A:1012238224409.

Kortekaas, F. (2007). HRM, organizational performance and the role of firm size (Master's thesis). Erasmus School of Economics, Rotterdam.

Kreps, D., \& Spence, M. (1985). Modelling the role of history in industrial organization and competition. In G. Feiwel (Eds.), Issues in contemporary microeconomics and welfare. London: Macmillan. DOI: 10.1007/978-1-349-06876-0_10.

Lenaghan, J.A., \& Eisner, A.B. (2006). Employers of choice and competitive advantage: The proof of the pudding is in the eating. Journal of Organizational Culture, Communications and Conflict, 10(1), 99-109.

Liu, W. (2004). The cross national transfer of HRM practices in MNCs: An integrative research model. International Journal of Manpower, 25(6), 500-517. DOI: 10.1108/01437720410560415.

Mincer, J. (1962). On the Job Training: Costs, Returns, and Some Implications. Journal of Political Economy, 70(5), 50-79. Recuperado de https://www.journals.uchicago.edu/doi/abs/10.1086/258725?journalCode=jpe

Morgan, J. (2014). The future of work. Attract new talent, build better leaders, and create a competitive organization. Hoboken, NJ: Wiley.

Nijhof, W., De-Jong, M., \& Beukhof, G. (1998). Employee commitment in changing organizations: an exploration. Journal of European Industrial Training, 22(6), 243-248. DOI: $10.1108 / 03090599810224701$.

OCDE (1991). Employment Outlook. Julio. Paris: OCDE. Recuperado de http://www.oecd.org/els/employmentoutlook-previouseditions.htm

Osterman, P. (1994). How Common Is Workplace Transformation and Who Adopts It? Industrial 
and Labor Relations Review, 47(2), 173-188. DOI: 10.1177\%2F001979399404700202

Paawee, J., \& Boselie, P. (2007). HRM and societal embeddedness. En P. Boxall, J. Purcell y P. Wright (Eds.), The Oxford Handbook of Human Resource Management (pp. 166-184). Oxford: Oxford University Press.

Romero, E.J. (2004). Are the great places to work also great performers? Academy of Management Perspectives, 18(2), 150-152. DOI: 10.5465/AME.2004.13835923.

Smith, A. (1993). Training and Enterprise Performance: Is There any Link? Training Agenda, 1(3), 24-27.

Snyder, C.R., \& Lopez, S.J. (2002). Handbook of positive psychology. New York: Oxford University Press.

Wright, P.M., McMahan, G.C., \& McWilliams, A. (1994). Human resources and sustained competitive advantage: a resource-based perspective. International Journal of Human Resource Management, 5, 301-326. DOI: 10.1080/09585199400000020. 